\title{
On some properties of a system of nonlinear partial functional differential equations
}

\author{
László Simon ${ }^{凶}$ \\ Eötvös Loránd University, Pázmány P. sétány 1/C, Budapest, H-1117, Hungary \\ Appeared 11 August 2016 \\ Communicated by Tibor Krisztin
}

\begin{abstract}
We consider a system of a semilinear hyperbolic functional differential equation (where the lower order terms contain functional dependence on the unknown function) with initial and boundary conditions and a quasilinear elliptic functional differential equation (containing $t$ as a parameter) with boundary conditions. Existence and some qualitative properties of weak solutions for $t \in(0, \infty)$ are proved.
\end{abstract}

Keywords: partial functional-differential equations, nonlinear systems of partial differential equations, nonlinear systems of mixed type, qualitative properties.

2010 Mathematics Subject Classification: 35M33, 35R10.

\section{Introduction}

In the present paper we consider weak solutions of the following system of equations:

$$
\begin{gathered}
u^{\prime \prime}(t)+Q(u(t))+\varphi(x) h^{\prime}(u(t))+H(t, x ; u, z)+\psi(x) u^{\prime}(t)=F_{1}(t, x ; z), \\
-\sum_{j=1}^{n} D_{j}\left[a_{j}(t, x, D z(t), z(t) ; u)\right]+a_{0}(t, x, D z(t), z(t) ; u, z)=F_{2}(t, x ; u), \\
(t, x) \in Q_{T}=(0, T) \times \Omega
\end{gathered}
$$

where $\Omega \subset \mathbb{R}^{n}$ is a bounded domain and we use the notations $u(t)=u(t, x), u^{\prime}=D_{t} u, u^{\prime \prime}=$ $D_{t}^{2} u, z(t)=z(t, x), D z=\left(\frac{\partial z}{\partial x_{1}}, \ldots \frac{\partial z}{\partial x_{n}}\right), Q$ may be e.g. a linear second order symmetric elliptic differential operator in the variable $x ; h$ is a $C^{2}$ function having certain polynomial growth, $H$ contains nonlinear functional (non-local) dependence on $u$ and $z$, with some polynomial growth and $F_{1}$ contains some functional dependence on $z$. Further, the functions $a_{j}$ define a quasilinear elliptic differential operator in $x$ (for fixed $t$ ) with functional dependence on $u$ for $i=1, \ldots, n$ and on $u, z$ for $i=0$, respectively. Finally, $F_{2}$ may non-locally depend on $u$. The system (1.1), (1.2) consists of a semilinear hyperbolic functional equation and an elliptic functional equation (containing the time $t$ as a parameter).

This paper was motivated by some problems which were modelled by systems consisting of (functional) differential equations of different types. In [4] S. Cinca investigated a

\footnotetext{
$\bowtie$ Email: simonl@cs.elte.hu
} 
model, consisting of an elliptic, a parabolic and an ordinary nonlinear differential equation, which arise when modelling diffusion and transport in porous media with variable porosity. In [6] J. D. Logan, M. R. Petersen and T. S. Shores considered and numerically studied a similar system which describes reaction-mineralogy-porosity changes in porous media with one-dimensional space variable. J. H. Merkin, D. J. Needham and B. D. Sleeman considered in [7] a system, consisting of a nonlinear parabolic and an ordinary differential equation, as a mathematical model for the spread of morphogens with density dependent chemosensitivity. In $[3,8,9]$ the existence of solutions of such systems were studied.

In [12] existence of weak solutions was proved for $t \in(0, T)$. In this paper existence and some qualitative properties of weak solutions for $t \in(0, \infty)$ are proved.

In Section 2 the existence theorem in $(0, T)$ will be formulated and in Section 3 we shall prove existence and certain properties of solutions for $t \in(0, \infty)$.

\section{Solutions in $(0, T)$}

Denote by $\Omega \subset \mathbb{R}^{n}$ a bounded domain having the uniform $C^{1}$ regularity property (see [1]), $Q_{T}=(0, T) \times \Omega$. Denote by $W^{1, p}(\Omega)$ the Sobolev space of real valued functions with the norm

$$
\|u\|=\left[\int_{\Omega}\left(\sum_{j=1}^{n}\left|D_{j} u\right|^{p}+|u|^{p}\right) d x\right]^{1 / p} \quad\left(2 \leq p<\infty, \quad D_{j} u=\frac{\partial u}{\partial x_{j}}\right) .
$$

The number $q$ is defined by $1 / p+1 / q=1$. Further, let $V_{1} \subset W^{1,2}(\Omega)$ and $V_{2} \subset W^{1, p}(\Omega)$ be closed linear subspaces containing $C_{0}^{\infty}(\Omega), V_{j}^{\star}$ the dual spaces of $V_{j}$, the duality between $V_{j}^{\star}$ and $V_{j}$ will be denoted by $\langle\cdot, \cdot\rangle$, the scalar product in $L^{2}(\Omega)$ will be denoted by $(\cdot, \cdot)$. Finally, denote by $L^{p}\left(0, T ; V_{j}\right)$ the Banach space of the set of measurable functions $u:(0, T) \rightarrow V_{j}$ with the norm

$$
\|u\|_{L^{p}\left(0, T ; V_{j}\right)}=\left[\int_{0}^{T}\|u(t)\|_{V_{j}}^{p} d t\right]^{1 / p}
$$

and $L^{\infty}\left(0, T ; V_{j}\right), L^{\infty}\left(0, T ; L^{2}(\Omega)\right)$ the set of measurable functions $u:(0, T) \rightarrow V_{j}, u:(0, T) \rightarrow$ $L^{2}(\Omega)$, respectively, with the $L^{\infty}(0, T)$ norm of the functions $t \mapsto\|u(t)\|_{V_{j}}, t \mapsto\|u(t)\|_{L^{2}(\Omega)}$, respectively.

First we formulate the existence theorem for $t \in(0, T)$ which was proved in [12], by using the results of [11], the theory of monotone operators (see, e.g., $[14,15])$ and Schauder's fixed point theorem.

Now we formulate the assumptions on the functions in (1.1), (1.2).

$\left(A_{1}\right) Q: V_{1} \rightarrow V_{1}^{\star}$ is a linear continuous operator such that

$$
\langle Q u, v\rangle=\langle Q v, u\rangle, \quad\langle Q u, u\rangle \geq c_{0}\|u\|_{V_{1}}^{2}
$$

for all $u, v \in V_{1}$ with some constant $c_{0}>0$.

$\left(A_{2}\right) \varphi, \psi: \Omega \rightarrow \mathbb{R}$ are measurable functions satisfying

$$
c_{1} \leq \varphi(x) \leq c_{2}, \quad c_{1} \leq \psi(x) \leq c_{2} \quad \text { for a.a. } x \in \Omega
$$

with some positive constants $c_{1}, c_{2}$. 
$\left(A_{3}\right) h: \mathbb{R} \rightarrow \mathbb{R}$ is a twice continuously differentiable function satisfying

$$
\begin{aligned}
& h(\eta) \geq 0, \quad\left|h^{\prime \prime}(\eta)\right| \leq \text { const }|\eta|^{\lambda-1} \quad \text { for }|\eta|>1 \text { where } \\
& 1<\lambda \leq \lambda_{0}=\frac{n}{n-2} \quad \text { if } n \geq 3, \quad 1<\lambda<\infty \quad \text { if } n=2 .
\end{aligned}
$$

$\left(A_{4}\right) H: Q_{T} \times L^{2}\left(Q_{T}\right) \times L^{p}\left(0, T ; V_{2}\right) \rightarrow \mathbb{R}$ is a function for which $(t, x) \mapsto H(t, x ; u, z)$ is measurable for all fixed $u \in L^{2}(\Omega), z \in L^{p}\left(0, T ; V_{2}\right), H$ has the Volterra property, i.e. for all $t \in[0, T], H(t, x ; u, z)$ depends only on the restriction of $u$ and $z$ to $(0, t)$. Further, the following inequality holds for all $t \in[0, T]$ and $u, u_{j} \in L^{2}(\Omega), z \in L^{p}\left(0, T ; V_{2}\right)$ :

$$
\begin{gathered}
\int_{\Omega}|H(t, x ; u, z)|^{2} d x \leq \text { const }\left[\|z\|_{L^{p}\left(0, T ; V_{2}\right)}^{2}+1\right]\left[\int_{0}^{t} \int_{\Omega} h(u) d x d \tau+\int_{\Omega} h(u) d x+1\right] ; \\
\int_{0}^{t}\left[\int_{\Omega}\left|H\left(\tau, x ; u_{1}, z\right)-H\left(\tau, x ; u_{2}, z\right)\right|^{2} d x\right] d \tau \leq M(K, z) \int_{0}^{t}\left[\int_{\Omega}\left|u_{1}-u_{2}\right|^{2} d x\right] d \tau \\
\text { if }\left\|u_{j}\right\|_{L^{\infty}\left(0, T ; V_{1}\right)} \leq K
\end{gathered}
$$

where for all fixed number $K>0, z \mapsto M(K, z) \in \mathbb{R}^{+}$is a bounded (nonlinear) operator. Finally, $\left(z_{k}\right) \rightarrow z$ in $L^{p}\left(0, T ; V_{2}\right)$ implies

$$
H\left(t, x ; u_{k}, z_{k}\right)-H\left(t, x ; u_{k}, z\right) \rightarrow 0 \text { in } L^{2}\left(Q_{T}\right) \text { uniformly if }\left\|u_{k}\right\|_{L^{2}\left(Q_{T}\right)} \leq \text { const. }
$$

$\left(A_{5}\right) F_{1}: Q_{T} \times L^{p}\left(0, T ; V_{2}\right) \rightarrow \mathbb{R}$ is a function satisfying $(t, x) \mapsto F_{1}(t, x ; z) \in L^{2}\left(Q_{T}\right)$ for all fixed $z \in L^{p}\left(0, T ; V_{2}\right)$ and $\left(z_{k}\right) \rightarrow z$ in $L^{p}\left(0, T ; V_{2}\right)$ implies that $F_{1}\left(t, x ; z_{k}\right) \rightarrow F_{1}(t, x ; z)$ in $L^{2}\left(Q_{T}\right)$.

Further,

$$
\int_{0}^{T}\left\|F_{1}(\tau, x ; z)\right\|_{L^{2}(\Omega)}^{2} d \tau \leq \mathrm{const}\left[1+\|z\|_{L^{p}\left(0, T ; V_{2}\right)}^{\beta_{1}}\right]
$$

with some constant $\beta_{1}>0$.

$\left(B_{1}\right)$ The functions

$$
\begin{aligned}
& a_{j}: Q_{T} \times \mathbb{R}^{n+1} \times L^{2}\left(Q_{T}\right) \rightarrow \mathbb{R} \quad(j=1, \ldots n), \\
& a_{0}: Q_{T} \times \mathbb{R}^{n+1} \times L^{2}\left(Q_{T}\right) \times L^{p}\left(0, T ; V_{2}\right) \rightarrow \mathbb{R}
\end{aligned}
$$

are such that $a_{j}(t, x, \xi ; u), a_{0}(t, x, \xi ; u, z)$ are measurable functions of variable $(t, x) \in Q_{T}$ for all fixed $\xi \in \mathbb{R}^{n+1}, u \in L^{2}\left(Q_{T}\right), z \in L^{p}\left(0, T ; V_{2}\right)$ and continuous functions of variable $\xi \in \mathbb{R}^{n+1}$ for all fixed $u \in L^{2}\left(Q_{T}\right), z \in L^{p}\left(0, T ; V_{2}\right)$ and a.a. fixed $(t, x) \in Q_{T}$.

Further, if $\left(u_{k}\right) \rightarrow u$ in $L^{2}\left(Q_{T}\right)$ then for all $z \in L^{p}\left(0, T ; V_{2}\right), \xi \in \mathbb{R}^{n+1}$, a.a. $(t, x) \in Q_{T}$, for a subsequence

$$
\begin{gathered}
a_{j}\left(t, x, \xi ; u_{k}\right) \rightarrow a_{j}(t, x, \xi ; u) \quad(j=1, \ldots, n), \\
a_{0}\left(t, x, \xi ; u_{k}, z_{k}\right)-a_{0}\left(t, x, \xi ; u, z_{k}\right) \rightarrow 0 .
\end{gathered}
$$

$\left(B_{2}\right)$ For $j=1, \ldots, n$

$$
\left|a_{j}(t, x, \xi ; u)\right| \leq g_{1}(u)|\xi|^{p-1}+\left[k_{1}(u)\right](t, x),
$$

where $g_{1}: L^{2}\left(Q_{T}\right) \rightarrow \mathbb{R}^{+}$is a bounded, continuous (nonlinear) operator,

$$
k_{1}: L^{2}\left(Q_{T}\right) \rightarrow L^{q}\left(Q_{T}\right) \text { is continuous and }\left\|k_{1}(u)\right\|_{L^{q}\left(Q_{T}\right)} \leq \operatorname{const}\left(1+\|u\|_{L^{2}\left(Q_{T}\right)}^{\gamma}\right) ;
$$




$$
\left|a_{0}(t, x, \xi ; u, z)\right| \leq g_{2}(u, z)|\xi|^{p-1}+\left[k_{2}(u, z)\right](t, x)
$$

where

$$
g_{2}: L^{2}\left(Q_{T}\right) \times L^{p}\left(0, T ; V_{2}\right) \rightarrow \mathbb{R}^{+} \quad \text { and } \quad k_{2}: L^{2}\left(Q_{T}\right) \times L^{p}\left(0, T ; V_{2}\right) \rightarrow L^{q}\left(Q_{T}\right)
$$

are continuous bounded operators such that

$$
\left\|k_{2}(u, z)\right\|_{L^{q}\left(Q_{T}\right)} \leq \text { const }\left[1+\|u\|_{L^{2}\left(Q_{T}\right)}^{\gamma}\right]
$$

with some constant $\gamma>0$.

$\left(B_{3}\right)$ The following inequality holds for all $t \in[0, T]$ with some constants $c_{2}>0, \beta>0$ (not depending on $t, u$ ):

$$
\begin{aligned}
& \int_{Q_{T}} \sum_{j=1}^{n}\left[a_{j}(t, x, D z(t), z(t) ; u)-a_{j}\left(t, x, D z^{\star}(t), z^{\star}(t) ; u\right)\right]\left[D_{j} z(t)-D_{j} z^{\star}(t)\right] d x d t \\
& +\int_{Q_{T}}\left[a_{0}(t, x, D z(t), z(t) ; u, z)-a_{0}\left(t, x, D z^{\star}(t), z^{\star}(t) ; u, z^{\star}\right)\right]\left[z(t)-z^{\star}(t)\right] d x d t \\
& \quad \geq \frac{c_{2}}{1+\|u\|_{L^{2}\left(Q_{T}\right)}^{\beta}}\left\|z-z^{\star}\right\|_{L^{p}\left(0, T ; V_{2}\right)}^{p} .
\end{aligned}
$$

$\left(B_{4}\right)$ For all fixed $u \in L^{2}\left(Q_{T}\right)$ the function

$\left(\right.$ see $\left.\left(B_{2}\right)\right)$ and

$$
\begin{gathered}
F_{2}: Q_{T} \times L^{2}\left(Q_{T}\right) \rightarrow \mathbb{R} \text { satisfies }(t, x) \mapsto F_{2}(t, x ; u) \in L^{q}\left(Q_{T}\right), \\
\left\|F_{2}(t, x ; u)\right\|_{L^{q}\left(Q_{T}\right)} \leq \mathrm{const}\left[1+\|u\|_{L^{2}\left(Q_{T}\right)}^{\gamma}\right]
\end{gathered}
$$

$$
\left(u_{k}\right) \rightarrow u \text { in } L^{2}\left(Q_{T}\right) \text { implies } F_{2}\left(t, x ; u_{k}\right) \rightarrow F_{2}(t, x ; u) \text { in } L^{q}\left(Q_{T}\right) .
$$

Finally,

$$
\frac{\beta_{1}}{2} \frac{\beta+\gamma}{p-1}<1
$$

Theorem 2.1. Assume $\left(A_{1}\right)-\left(A_{5}\right)$ and $\left(B_{1}\right)-\left(B_{4}\right)$. Then for all $u_{0} \in V_{1}, u_{1} \in L^{2}(\Omega)$ there exists $u \in L^{\infty}\left(0, T ; V_{1}\right)$ such that

$$
u^{\prime} \in L^{\infty}\left(0, T ; L^{2}(\Omega)\right), \quad u^{\prime \prime} \in L^{2}\left(0, T ; V_{1}^{\star}\right) \quad \text { and } \quad z \in L^{p}\left(0, T ; V_{2}\right)
$$

such that $u, z$ satisfy (1.1) in the sense: for a.a. $t \in[0, T]$, all $v \in V_{1}$

$$
\begin{aligned}
& \left\langle u^{\prime \prime}(t), v\right\rangle+\langle Q(u(t)), v\rangle+\int_{\Omega} \varphi(x) h^{\prime}(u(t)) v d x+\int_{\Omega} H(t, x ; u, z) v d x+\int_{\Omega} \psi(x) u^{\prime}(t) v d x \\
& \left.\quad=\int_{\Omega} F_{1}(t, x ; z) v\right) d x
\end{aligned}
$$

and the initial conditions

$$
u(0)=u_{0}, \quad u^{\prime}(0)=u_{1} .
$$

Further, $u, z$ satisfy (1.2) in the sense: for a.a. $t \in(0, T)$, all $w \in V_{2}$

$$
\begin{aligned}
\int_{\Omega}\left[\sum_{j=1}^{n} a_{j}(t, x, D z(t), z(t) ; u)\right] D_{j} w d x+\int_{\Omega} a_{0}(t, x, D z(t), z(t) ; u, z) w d x \\
\quad=\int_{\Omega} F_{2}(t, x ; u) w d x .
\end{aligned}
$$

Remark 2.2. Examples, satisfying the assumptions of Theorem 2.1 can be found in [12]. 


\section{Main steps of the proof}

Now we formulate the main steps in the proof in Theorem 2.1 which will be applied in the next section. (For the detailed proof, see [12].)

Consider the problem (2.1), (2.2) for $u$ with an arbitrary fixed $z=\tilde{z} \in L^{p}\left(0, T ; V_{2}\right)$. According to [11] assumptions $\left(A_{1}\right)-\left(A_{5}\right)$ imply that there exists a unique solution $u=\tilde{u} \in$ $L^{\infty}\left(0, T ; V_{1}\right)$ with the properties $\tilde{u}^{\prime} \in L^{\infty}\left(0, T ; L^{2}(\Omega)\right), \tilde{u}^{\prime \prime} \in L^{2}\left(0, T ; V_{1}^{\star}\right)$ satisfying (2.1) and the initial condition (2.2). Then consider problem (2.3) for $z$ with the above $u=\tilde{u}$. According to the theory of monotone operators (see, e.g., $[14,15])$ there exists a unique solution $z \in L^{p}\left(0, T ; V_{2}\right)$ of (2.3). By using the notation $S(\tilde{z})=z$, it is shown that the operator $S: L^{p}\left(0, T ; V_{2}\right) \rightarrow L^{p}\left(0, T ; V_{2}\right)$ satisfies the assumptions of Schauder's fixed point theorem: it is continuous, compact and there exists a closed ball $B_{0}(R) \subset L^{p}\left(0, T ; V_{2}\right)$ such that

$$
S\left(B_{0}(R)\right) \subset B_{0}(R) .
$$

Then Schauder's fixed point theorem implies that $S$ has a fixed point $z^{\star} \in L^{p}\left(0, T ; V_{2}\right)$. Defining $u^{\star}$ by the solution of (2.1), (2.2) with $z=z^{\star}$, functions $u^{\star}, z^{\star}$ satisfy (2.1)-(2.3).

Now we formulate some details of the proof which will be used in the next section.

According to [11] the solution $\tilde{u}$ of $(2.1),(2.2)$ with $z=\tilde{z}$ we obtain as the weak limit in $L^{p}\left(0, T ; V_{1}\right)$ of Galerkin approximations

$$
\tilde{u}_{m}(t)=\sum_{l=1}^{m} g_{l m}(t) w_{l} \quad \text { where } \quad g_{l m} \in W^{2,2}(0, T)
$$

and $w_{1}, w_{2}, \ldots$ is a linearly independent system in $V_{1}$ such that the linear combinations are dense in $V_{1}$, further, the functions $\tilde{u}_{m}$ satisfy (for $j=1, \ldots, m$ )

$$
\begin{gathered}
\left\langle\tilde{u}_{m}^{\prime \prime}(t), w_{j}\right\rangle+\left\langle Q\left(\tilde{u}_{m}(t)\right), w_{j}\right\rangle+\int_{\Omega} \varphi(x) h^{\prime}\left(\tilde{u}_{m}(t)\right) w_{j} d x \\
+\int_{\Omega} H\left(t, x ; \tilde{u}_{m}, \tilde{z}\right) w_{j} d x+\int_{\Omega} \psi(x) \tilde{u}_{m}^{\prime}(t) w_{j} d x=\int_{\Omega} F_{1}(t, x ; \tilde{z}) w_{j} d x, \\
\tilde{u}_{m}(0)=u_{m 0}, \quad \tilde{u}_{m}^{\prime}(0)=u_{m 1}
\end{gathered}
$$

where $u_{m 0}, u_{m 1}(m=1,2, \ldots)$ are linear combinations of $w_{1}, w_{2}, \ldots, w_{m}$, satisfying $\left(u_{m 0}\right) \rightarrow u_{0}$ in $V_{1}$ and $\left(u_{m 1}\right) \rightarrow u_{1}$ in $L^{2}(\Omega)$ as $m \rightarrow \infty$.

Multiplying (2.5) by $\left(g_{j m}\right)^{\prime}(t)$, summing with respect to $j$ and integrating over $(0, t)$, by Young's inequality we find

$$
\begin{aligned}
& \frac{1}{2}\left\|\tilde{u}_{m}^{\prime}(t)\right\|_{L^{2}(\Omega)}^{2}+\frac{1}{2}\left\langle Q\left(\tilde{u}_{m}(t)\right), \tilde{u}_{m}(t)\right\rangle+\int_{\Omega} \varphi(x) h\left(\tilde{u}_{m}(t)\right) d x \\
& +\int_{0}^{t}\left[\int_{\Omega} H\left(\tau, x ; \tilde{u}_{m}, \tilde{z}_{k}\right) \tilde{u}_{m}^{\prime}(\tau) d x\right] d \tau+\int_{0}^{t}\left[\int_{\Omega} \psi(x)\left|\tilde{u}_{m}^{\prime}(\tau)\right|^{2} d x\right] d \tau \\
& =\int_{0}^{t}\left[\int_{\Omega} F_{1}(\tau, x ; \tilde{z}) \tilde{u}_{m}^{\prime}(\tau) d x\right] d \tau+\frac{1}{2}\left\|\tilde{u}_{m}^{\prime}(0)\right\|_{L^{2}(\Omega)}^{2}+\frac{1}{2}\left\langle Q\left(\tilde{u}_{m}(0)\right), \tilde{u}_{m}(0)\right\rangle \\
& \quad+\int_{\Omega} \varphi(x) h\left(\tilde{u}_{m}(0)\right) d x \leq \frac{1}{2} \int_{0}^{T}\left\|F_{1}(\tau, x ; \tilde{z})\right\|_{L^{2}(\Omega)}^{2} d \tau+\frac{1}{2} \int_{0}^{T}\left\|\tilde{u}_{m}^{\prime}(\tau)\right\|_{L^{2}(\Omega)}^{2}+\text { const }
\end{aligned}
$$


where the constant is not depending on $m, k, t$. (See [11].)

By using $\left(A_{2}\right),\left(A_{4}\right),\left(A_{5}\right)$ and the Cauchy-Schwarz inequality, we obtain from (2.7)

$$
\begin{aligned}
& \frac{1}{2}\left\|\tilde{u}_{m}^{\prime}(t)\right\|_{L^{2}(\Omega)}^{2} d \tau+{ }^{2}+\frac{c_{0}}{2}\left\|\tilde{u}_{m}(t)\right\|_{V_{1}}^{2}+c_{1} \int_{\Omega} h\left(\tilde{u}_{m}(t)\right) d x \\
& \quad \leq \int_{0}^{T}\left\|F_{1}(\tau, x ; \tilde{z})\right\|_{L^{2}(\Omega)}^{2} d \tau+\operatorname{const}\left\{1+\int_{0}^{t}\left\|\tilde{u}_{m}^{\prime}(\tau)\right\|_{L^{2}(\Omega)}^{2} d \tau+\int_{0}^{t}\left[\int_{\Omega} h\left(\tilde{u}_{m}(\tau)\right) d x\right] d \tau\right\}
\end{aligned}
$$

where the constants are not depending on $m, t, \tilde{z}$. Hence, by Gronwall's lemma one obtains

$$
\begin{aligned}
& \left\|\tilde{u}_{m}^{\prime}(t)\right\|_{L^{2}(\Omega)}^{2}+\int_{\Omega} h\left(\tilde{u}_{m}(t)\right) d x \\
& \quad \leq \operatorname{const} \int_{0}^{T}\left\|F_{1}(\tau, x ; \tilde{z})\right\|_{L^{2}(\Omega)}^{2} d \tau+\text { const } \int_{0}^{t}\left[\int_{0}^{T}\left[1+\left\|F_{1}(\tau, x ; \tilde{z})\right\|_{L^{2}(\Omega)}^{2} d \tau\right] \cdot \mathrm{e}^{t-s}\right] d s \\
& \quad=\operatorname{const} \int_{0}^{T}\left\|F_{1}(\tau, x ; \tilde{z})\right\|_{L^{2}(\Omega)}^{2} d \tau
\end{aligned}
$$

where the constants are independent of $m, t, \tilde{z}$. Thus by (2.8) and $\left(A_{5}\right)$ we find

$$
\left\|\tilde{u}_{m}(t)\right\|_{V_{1}}^{2} \leq \mathrm{const} \int_{0}^{T}\left\|F_{1}(\tau, x ; \tilde{z})\right\|_{L^{2}(\Omega)}^{2} d \tau \leq \mathrm{const}\left[1+\|\tilde{z}\|_{L^{p}\left(0, T ; V_{2}\right)}^{\beta_{1}}\right]
$$

which implies (for the limit of $\left(\tilde{u}_{m}\right)$ )

$$
\|\tilde{u}\|_{L^{2}\left(Q_{T}\right)}^{2} \leq \text { const }\left[1+\|\tilde{z}\|_{L^{p}\left(0, T ; V_{2}\right)}^{\beta_{1}}\right] .
$$

On the other hand, by $\left(B_{3}\right),\left(B_{4}\right)$ we have for the solution $z$ of (2.3) with $u=\tilde{u}$

$$
\begin{aligned}
& \frac{c_{2}}{1+\|\tilde{u}\|_{L^{2}\left(Q_{T}\right)}^{\beta}}\|z\|_{L^{p}\left(0, T ; V_{2}\right)}^{p} \\
& \quad \leq\left\|F_{2}(t, x ; \tilde{u})\right\|_{L^{2}\left(Q_{T}\right)}\|z\|_{L^{p}\left(0, T ; V_{2}\right)}+\text { const }\left[\left\|k_{1}(\tilde{u})\right\|_{L^{q}\left(Q_{T}\right)}+c(\tilde{u})\right]\|z\|_{L^{p}\left(0, T ; V_{2}\right)}
\end{aligned}
$$

where the constant is not depending on $\tilde{u}$, further, by $\left(B_{2}\right)$

$$
\left\|k_{1}(\tilde{u})\right\|_{L^{q}\left(Q_{T}\right)} \leq \mathrm{const}\left[1+\|\tilde{u}\|_{L^{2}\left(Q_{T}\right)}^{\gamma}\right] \quad \text { and } \quad c(\tilde{u}) \leq \text { const }\left[1+\|\tilde{u}\|_{L^{2}\left(Q_{T}\right)}^{\gamma}\right] .
$$

The inequalities (2.11), (2.12) imply

$$
\|z\|_{L^{p}\left(0, T ; V_{2}\right)}^{p-1} \leq \mathrm{const}\left[1+\|\tilde{u}\|_{L^{2}\left(Q_{T}\right)}^{\beta}\right] \cdot\left[\left\|F_{2}(t, x ; \tilde{u})\right\|_{L^{2}\left(Q_{T}\right)}+1+\|\tilde{u}\|_{L^{2}\left(Q_{T}\right)}^{\gamma}\right]
$$

thus by $(2.10)$ and $\left(B_{4}\right)$

$$
\|z\|_{L^{p}\left(0, T ; V_{2}\right)} \leq \mathrm{const}\left[1+\|\tilde{u}\|_{L^{2}\left(Q_{T}\right)}^{\frac{\beta+\gamma}{p-1}}\right] \leq \mathrm{const}\left[1+\|\tilde{z}\|_{L^{p}\left(0, T ; V_{2}\right)}^{\frac{\beta_{1}(\beta+\gamma)}{2(p-1)}}\right]
$$

where the constants are not depending on $\tilde{u}$ and $\tilde{z}$.

According to the assumption $\left(B_{4}\right)$

$$
\frac{\beta_{1}(\beta+\gamma)}{2(p-1)}<1
$$

so (2.14) implies that there is a closed ball $B_{0}(R) \subset L^{p}\left(0, T ; V_{2}\right)$ such that $S\left(B_{0}(R)\right) \subset B_{0}(R)$. 


\section{Solutions in $(0, \infty)$}

Now we formulate an existence theorem with respect to solutions for $t \in(0, \infty)$. Denote by $L_{\text {loc }}^{p}\left(0, \infty ; V_{1}\right)$ the set of functions $u:(0, \infty) \rightarrow V_{1}$ such that for each fixed finite $T>0$, their restrictions to $(0, T)$ satisfy $\left.u\right|_{(0, T)} \in L^{p}\left(0, T ; V_{1}\right)$ and let $Q_{\infty}=(0, \infty) \times \Omega, L_{\text {loc }}^{\alpha}\left(Q_{\infty}\right)$ the set of functions $u: Q_{\infty} \rightarrow \mathbb{R}$ such that $\left.u\right|_{Q_{T}} \in L^{\alpha}\left(Q_{T}\right)$ for any finite $T$.

Now we formulate assumptions on $H, F_{1}, a_{j}, F_{2}$.

$\left(\tilde{A}_{4}\right)$ The function $H: Q_{\infty} \times L_{\text {loc }}^{2}\left(Q_{\infty}\right) \times L_{\text {loc }}^{p}\left(0, \infty ; V_{2}\right) \rightarrow \mathbb{R}$ is such that for all fixed $u \in$ $L_{\text {loc }}^{2}\left(Q_{\infty}\right), z \in L_{l o c}^{p}\left(0, \infty ; V_{2}\right)$ the function $(t, x) \mapsto H(t, x ; u, z)$ is measurable, $H$ has the Volterra property (see $\left(A_{4}\right)$ ) and for each fixed finite $T>0$, the restriction $H_{T}$ of $H$ to $Q_{T} \times L^{2}\left(Q_{T}\right) \times L^{p}\left(0, T ; V_{2}\right)$ satisfies $\left(A_{4}\right)$.

Remark 3.1. Since $H$ has the Volterra property, this restriction $H_{T}$ is well defined by the formula

$$
H_{T}(t, x ; \tilde{u}, \tilde{z})=H(t, x ; u, z), \quad(t, x) \in Q_{T}, \quad \tilde{u} \in L^{2}\left(Q_{T}\right), \quad \tilde{z} \in L^{p}\left(0, T ; V_{2}\right)
$$

where $u \in L_{\text {loc }}^{2}\left(Q_{\infty}\right), z \in L_{\text {loc }}^{p}\left(0, \infty ; V_{2}\right)$ may be any functions satisfying $u(t, x)=\tilde{u}(t, x)$, $z(t, x)=\tilde{z}(t, x)$ for $(t, x) \in Q_{T}$.

$\left(\tilde{A}_{5}\right) F_{1}: Q_{\infty} \times L_{\text {loc }}^{p}\left(0, \infty ; V_{2}\right) \rightarrow \mathbb{R}$ has the Volterra property and for each fixed finite $T>0$, the restriction of $F_{1}$ to $(0, T)$ satisfies $\left(A_{5}\right)$.

$(\tilde{B}) a_{j}: Q_{\infty} \times \mathbb{R}^{n+1} \times L_{\text {loc }}^{2}\left(Q_{\infty}\right) \rightarrow \mathbb{R}(j=1, \ldots, n)$ and $a_{0}: Q_{\infty} \times \mathbb{R}^{n+1} \times L_{\text {loc }}^{2}\left(Q_{\infty}\right) \times$ $L_{\text {loc }}^{p}\left(0, \infty ; V_{2}\right) \rightarrow \mathbb{R}$ have the Volterra property and for each finite $T>0$, their restrictions to $(0, T)$ satisfy $\left(B_{1}\right)-\left(B_{3}\right)$.

$\left(\tilde{B}_{4}\right) F_{2}: Q_{\infty} \times L_{\text {loc }}^{2}\left(Q_{\infty}\right) \rightarrow \mathbb{R}$ has the Volterra property and for each fixed finite $T>0$, the restriction of $F_{2}$ to $(0, T)$ satisfies $\left(B_{4}\right)$.

Theorem 3.2. Assume $\left(A_{1}\right)-\left(A_{3}\right),\left(\tilde{A}_{4}\right),\left(\tilde{A}_{5}\right),(\tilde{B}),\left(\tilde{B}_{4}\right)$. Then for all $u_{0} \in V_{1}, u_{1} \in L^{2}(\Omega)$ there exist

$$
\begin{array}{rlrl}
u & \in L_{\mathrm{loc}}^{\infty}\left(0, \infty ; V_{1}\right), & z & \in L_{\mathrm{loc}}^{p}\left(0, \infty ; V_{2}\right) \quad \text { such that } \\
u^{\prime} & \in L_{\mathrm{loc}}^{\infty}\left(0, \infty ; L^{2}(\Omega)\right), \quad u^{\prime \prime} \in L_{\mathrm{loc}}^{2}\left(0, \infty ; V_{1}^{\star}\right),
\end{array}
$$

(2.1) and (2.3) hold for a.a. $t \in(0, \infty)$ and the initial condition (2.2) is fulfilled.

Assume that the following additional conditions are satisfied: there exist $H^{\infty}, F_{1}^{\infty} \in L^{2}(\Omega), u_{\infty} \in$ $V_{1}$, a bounded function $\tilde{\beta}$, belonging to $L^{2}\left(0, \infty ; L^{2}(\Omega)\right)$ such that

$$
\begin{gathered}
Q\left(u_{\infty}\right)=F_{1}^{\infty}-H^{\infty}, \\
\left|H(t, x ; u, z)-H^{\infty}(x)\right| \leq \tilde{\beta}(t, x), \quad\left|F_{1}(t, x ; z)-F_{1}^{\infty}(x)\right| \leq \tilde{\beta}(t, x)
\end{gathered}
$$

for all fixed $\left.u \in L_{\mathrm{loc}}^{2}\left(Q_{\infty}\right), z \in L_{\mathrm{loc}}^{p}\left(0, \infty ; V_{2}\right)\right)$. Further, there exist functions

$$
\begin{array}{ll}
a_{j}^{\infty}: \Omega \times \mathbb{R}^{n+1} \times V_{1} \rightarrow \mathbb{R}, & j=1, \ldots, n \\
a_{0}^{\infty}: \Omega \times \mathbb{R}^{n+1} \times V_{1} \times V_{2} \rightarrow \mathbb{R}, & F_{2}^{\infty}: \Omega \times V_{1} \rightarrow \mathbb{R}
\end{array}
$$

such that for each fixed $z_{0} \in V_{2}$ and $w_{0} \in V_{1}$ with the property

$$
\lim _{t \rightarrow \infty}\left\|u(t)-w_{0}\right\|_{L^{2}(\Omega)}=0
$$




$$
\begin{gathered}
\lim _{t \rightarrow \infty}\left\|a_{j}\left(t, x, D z_{0}, z_{0} ; u\right)-a_{j}^{\infty}\left(x, D z_{0}, z_{0} ; w_{0}\right)\right\|_{L^{q}(\Omega)}=0, \quad j=1, \ldots, n, \\
\lim _{t \rightarrow \infty}\left\|a_{0}\left(t, x, D z_{0}, z_{0} ; u, z_{0}\right)-a_{0}^{\infty}\left(x, D z_{0}, z_{0} ; w_{0}, z_{0}\right)\right\|_{L^{q}(\Omega)}=0, \\
\lim _{t \rightarrow \infty}\left\|F_{2}(t, x ; u)-F_{2}^{\infty}\left(x ; w_{0}\right)\right\|_{L^{q}(\Omega)}=0 .
\end{gathered}
$$

Finally, $\left(B_{3}\right)$ is satisfied such that the following inequalities hold for all $t>0$ with some constants $c_{2}>0, \beta>0$ (not depending on $t$ ):

$$
\begin{aligned}
& \int_{\Omega} \sum_{j=1}^{n}\left[a_{j}(t, x, D z(t), z(t) ; u)-a_{j}\left(t, x, D z^{\star}(t), z^{\star}(t) ; u\right)\right]\left[D_{j} z-D_{j} z^{\star}\right] d x \\
& +\int_{\Omega}\left[a_{0}(t, x, D z(t), z(t) ; u, z)-a_{0}\left(t, x, D z^{\star}(t), z^{\star}(t) ; u, z^{\star}\right)\right]\left[z(t)-z^{\star}(t)\right] d x \\
& \quad \geq \frac{c_{2}}{1+\|u\|_{L^{2}\left(Q_{t} \backslash Q_{t-a}\right)}^{\beta}}\left\|z-z^{\star}\right\|_{V_{1}}^{p}
\end{aligned}
$$

with some fixed $a>0$ (finite delay).

Then for any solution $u, z$ of $(2.1)-(2.3)$ in $(0, \infty)$ we have

$$
\begin{gathered}
u \in L^{\infty}\left(0, \infty ; V_{1}\right), \\
\left\|u^{\prime}(t)\right\|_{H} \leq \text { const } \mathrm{e}^{-c_{1} T}
\end{gathered}
$$

where $c_{1}$ is given in $\left(A_{2}\right)$ and there exists $w_{0} \in V_{1}$ such that

$$
u(T) \rightarrow w_{0} \text { in } L^{2}(\Omega) \text { as } T \rightarrow \infty, \quad\left\|u(T)-w_{0}\right\|_{H} \leq \text { const } \mathrm{e}^{-c_{1} T}
$$

and $w_{0}$ satisfies

$$
Q\left(w_{0}\right)+\varphi h^{\prime}\left(w_{0}\right)=F_{1}^{\infty}-H^{\infty} .
$$

Finally, there exists a unique solution $z_{0} \in V_{2}$ of

$$
\begin{gathered}
\sum_{j=1}^{n} \int_{\Omega} a_{j}^{\infty}\left(x, D z_{0}, z_{0} ; w_{0}\right) D_{j} v d x+\int_{\Omega} a_{0}^{\infty}\left(x, D z_{0}, z_{0} ; w_{0}, z_{0}\right) v d x \\
\quad=\int_{\Omega} F_{2}^{\infty}\left(x ; w_{0}\right) v d x \quad \text { for all } v \in V_{2}
\end{gathered}
$$

(where $w_{0}$ is the solution of (3.10)) and

$$
\lim _{t \rightarrow \infty}\left\|z(t)-z_{0}\right\|_{V_{2}}=0
$$

Proof. Let $\left(T_{k}\right)_{k \in \mathbb{N}}$ be a monotone increasing sequence, converging to $+\infty$. According to Theorem 2.1, there exist solutions $u_{k}, z_{k}$ of (2.1)-(2.3) for $t \in\left(0, T_{k}\right)$. The Volterra property of $H$, $F_{1}, a_{j}, F_{2}$ implies that the restrictions of $u_{k}, z_{k}$ to $t \in\left(0, T_{l}\right)$ with $T_{l}<T_{k}$ satisfy (2.1)-(2.3) for $t \in\left(0, T_{l}\right)$.

Now consider the restrictions $\left.u_{k}\right|_{\left(0, T_{1}\right)},\left.z_{k}\right|_{\left(0, T_{1}\right)}, k=2,3, \ldots$ Applying (2.14) to $T=T_{1}$ and $z=\tilde{z}=\left.z_{k}\right|_{\left(0, T_{1}\right)}$, by (2.15) we obtain that the sequence

$$
\left(\left.z_{k}\right|_{\left(0, T_{1}\right)}\right)_{k \in \mathbb{N}} \text { is bounded in } L^{p}\left(0, T_{1} ; V_{2}\right) \text {. }
$$

The operator $S: L^{p}\left(0, T_{1} ; V_{2}\right) \rightarrow L^{p}\left(0, T_{1} ; V_{2}\right)$ is compact thus there is a subsequence $\left(z_{1 k}\right)_{k \in \mathbb{N}}$ of $\left(z_{k}\right)_{k \in \mathbb{N}}$ such that the sequence of restrictions $\left(\left.z_{1 k}\right|_{\left(0, T_{1}\right)}\right)_{k \in \mathbb{N}}$ is convergent in $L^{p}\left(0, T_{1} ; V_{2}\right)$. 
Now consider the restrictions $\left.z_{1 k}\right|_{\left(0, T_{2}\right)}$ By using the above arguments, we find that there exists a subsequence $\left(z_{2 k}\right)_{k \in \mathbb{N}}$ of $\left(z_{1 k}\right)_{k \in \mathbb{N}}$ such that $\left(\left.z_{2 k}\right|_{\left(0, T_{2}\right)}\right)_{k \in \mathbb{N}}$ is convergent in $L^{p}\left(0, T_{2} ; V_{2}\right)$.

Thus for all $l \in \mathbb{N}$ we obtain a subsequence $\left(z_{l k}\right)_{k \in \mathbb{N}}$ of $\left(z_{k}\right)_{k \in \mathbb{N}}$ such that $\left(\left.z_{l k}\right|_{\left(0, T_{l}\right)}\right)_{k \in \mathbb{N}}$ is convergent in $L^{p}\left(0, T_{l} ; V_{2}\right)$. Then the diagonal sequence $\left(z_{k k}\right)_{k \in \mathbb{N}}$ is a subsequence of $\left(z_{k}\right)_{k \in \mathbb{N}}$ such that for all fixed $l \in \mathbb{N},\left(\left.z_{k k}\right|_{\left(0, T_{l}\right)}\right)_{k \in \mathbb{N}}$ is convergent in $L^{p}\left(0, T_{l} ; V_{2}\right)$ to some $z^{\star} \in$ $L_{\text {loc }}^{p}\left(0, \infty ; V_{2}\right)$. Since $z_{l l}$ is a fixed point of $S=S_{l}: L^{p}\left(0, T_{l} ; V_{2}\right) \rightarrow L^{p}\left(0, T_{l} ; V_{2}\right)$ and $S_{l}$ is continuous thus the limit $\left.z^{\star}\right|_{\left(0, T_{l}\right)}$ in $L^{p}\left(0, T_{l} ; V_{2}\right)$ of $\left(\left.z_{k k}\right|_{\left(0, T_{l}\right)}\right)_{k \in \mathbb{N}}$ is a fixed point of $S=S_{l}$.

Consequently, the solutions $u_{l}^{\star}$ of (2.1), (2.2) when $z$ is the restriction of $z^{\star}$ to $\left(0, T_{l}\right)$ and the restriction of $z^{\star}$ to $\left(0, T_{l}\right)$ satisfy (2.1)-(2.3) for $t \in\left(0, T_{l}\right)$. Since for $m<l,\left.u_{l}^{\star}\right|_{\left(0, T_{m}\right)}=u_{m}^{\star}$ (by the Volterra property of $\left.H, F_{1}, a_{j}, F_{2}\right)$, we obtain $u^{\star} \in L_{l o c}^{2}\left(Q_{\infty}\right)$ such that for all fixed $l$, $\left.u^{\star}\right|_{\left(0, T_{l}\right)},\left.z^{\star}\right|_{\left(0, T_{l}\right)}$ satisfy (2.1)-(2.3) for $t \in\left(0, T_{l}\right)$, so the first part of Theorem 3.2 is proved.

Now assume that the additional conditions (3.1), (3.2) are satisfied. Then we obtain (3.7)(3.10) for $u=u^{\star}, z=z^{\star}$ by using the arguments of the proof of Theorem 3.2 in [11]. For convenience we formulate the main steps of the proof.

Let $u, z$ be arbitrary solutions of (2.1)-(2.3) for $t \in(0, \infty)$ and $z_{k k}=\left.z\right|_{\left(0, T_{k}\right)}, u_{k k}=\left.u\right|_{\left(0, T_{k}\right)}$. Then $z_{k k}, u_{k k}$ are solutions of (2.1)-(2.3) for $t \in\left(0, T_{l}\right)$ if $k \geq l$, hence the sequence $\left.\left(z_{k k}\right)\right|_{k \in \mathbb{N}}$ is bounded in $L^{p}\left(0, T_{l} ; V_{2}\right)$ for each fixed $l$ (see, e.g., (3.13)), consequently, from (2.7) (with $\tilde{z}_{k}=z_{k k}$ ) we obtain for the solutions $u_{k k}$ of (2.1), (2.2) with $\tilde{z}=z_{k k}$ (since $u_{k k}$ is the limit of the Galerkin approximations)

$$
\begin{aligned}
& \frac{1}{2}\left\|u_{k k}^{\prime}(t)\right\|_{H}^{2}+\frac{1}{2}\left\langle Q\left(u_{k k}(t)\right), u_{k k}(t)\right\rangle+\int_{\Omega} \varphi(x) h\left(u_{k k}(t)\right) d x \\
& +\int_{0}^{t}\left[\int_{\Omega} \psi(x)\left|u_{k k}^{\prime}(\tau)\right|^{2} d x\right] d \tau+\int_{0}^{t}\left[\int_{\Omega} H\left(\tau, x ; u_{k k}, z_{k k}\right) u_{k k}^{\prime}(\tau) d x\right] d \tau \\
& =\int_{0}^{t}\left[\int_{\Omega} F_{1}\left(\tau, x ; z_{k k}\right) u_{k k}^{\prime}(\tau) d x\right] d \tau+\frac{1}{2}\left\|u_{k k}^{\prime}(0)\right\|_{H}^{2}+\frac{1}{2}\left\langle Q\left(u_{k k}(0)\right), u_{k k}(t)\right\rangle \\
& \quad+\int_{\Omega} \varphi(x) h\left(u_{k k}(0)\right) d x
\end{aligned}
$$

for all $t>0$. Hence we find by (3.1), (3.2) and Young's inequality for $w_{k k}=u_{k k}-u_{\infty}$

$$
\begin{aligned}
& \left.\frac{1}{2}\left\|w_{k k}^{\prime}(t)\right\|_{L^{2}(\Omega)}^{2}+\frac{c_{0}}{2} \| u_{k k}(t)\right) \|_{V_{1}}^{2}+c_{1} \int_{\Omega} h\left(u_{k k}(t)\right) d x+\text { const } \int_{0}^{t}\left[\int_{\Omega}\left|w_{k k}^{\prime}\right|^{2} d x\right] d \tau \\
& \leq \operatorname{const}\left\{\int_{0}^{t}\left\|F_{1}\left(\tau, x ; z_{k k}\right)-F_{1}^{\infty}\right\|_{L^{2}(\Omega)}^{2} d \tau+\int_{0}^{t}\left\|H\left(\tau, x ; u_{k k} z_{k k}\right)-H^{\infty}\right\|_{L^{2}(\Omega)}^{2} d \tau\right\} \\
& \quad+\varepsilon \int_{0}^{t}\left[\int_{\Omega}\left|w_{k k}^{\prime}\right|^{2} d x\right] d \tau+\frac{1}{2}\left\|u_{k k}^{\prime}(0)\right\|_{L^{2}(\Omega)}^{2}+\frac{1}{2}\left\langle Q\left(u_{k k}(0)\right), u_{k k}(0)\right\rangle+c_{2} \int_{\Omega} h\left(u_{k k}(0)\right) d x \\
& \leq \varepsilon \int_{0}^{t}\left[\int_{\Omega}\left|w_{k k}^{\prime}\right|^{2} d x\right] d \tau+\text { const }+C(\varepsilon)\|\tilde{\beta}\|_{L^{2}\left(0, \infty ; L^{2}(\Omega)\right)}^{2} .
\end{aligned}
$$

Choosing sufficiently small $\varepsilon>0$, we obtain

$$
\int_{0}^{t}\left[\int_{\Omega}\left|w_{k k}^{\prime}\right|^{2} d x\right] d \tau \leq \text { const }
$$

and thus by (3.15)

$$
\left\|u_{k k}^{\prime}(t)\right\|_{L^{2}(\Omega)}^{2}+\tilde{c} \int_{0}^{t}\left\|u_{k k}^{\prime}(\tau)\right\|_{L^{2}(\Omega)}^{2} d \tau \leq c^{\star}
$$

with some positive constants $\tilde{c}$ and $c^{\star}$ not depending on $k$ and $t \in(0, \infty)$. Hence by Gronwall's lemma we obtain (3.8) for the weak limit of the sequence $\left(u_{k k}\right)$ and by (3.15) we find (3.7). 
It is not difficult to show that

$$
\left\|u\left(T_{2}\right)-u\left(T_{1}\right)\right\|_{L^{2}(\Omega)} \leq \int_{T_{1}}^{T_{2}}\left\|u^{\prime}(t)\right\|_{L^{2}(\Omega)} d t
$$

(see [11]), thus (3.8) implies (3.9) and by $u \in L^{\infty}\left(0, \infty ; V_{1}\right)$, the limit $w_{0}$ of $u(t)$ as $t \rightarrow \infty$ must belong to $V_{1}$.

In order to prove (3.10) we apply equation (1.1) to $v \chi_{T_{k}}(t)$ with arbitrary fixed $v \in V_{1}$ where $\lim _{k \rightarrow \infty}\left(T_{k}\right)=+\infty$ and

$$
\chi_{T_{k}}(t)=\chi\left(t-T_{k}\right), \quad \chi \in C_{0}^{\infty}, \quad \operatorname{supp} \chi \subset[0,1], \quad \int_{0}^{1} \chi(t) d t=1 .
$$

Then by (3.8) one obtains (3.10) as $k \rightarrow \infty$.

Now we show that there exists a unique solution $z_{0} \in V_{2}$ of (3.11). This statement follows from the fact that the operator (applied to $z_{0} \in V_{2}$ ) on the left-hand side of (3.11) is bounded, demicontinuous and uniformly monotone (see, e.g. [14,15]) by $\left(B_{1}\right),\left(B_{2}\right),(3.9)(3.3),(3.4),(3.6)$.

Finally, we show (3.12). By (3.6) we have

$$
\begin{aligned}
& \frac{c_{2}}{1+\|u\|_{L^{2}\left(Q_{t} \backslash Q_{t-a}\right)}}\left\|z(t)-z_{0}\right\|_{V_{2}}^{p} \\
& \leq \int_{\Omega} \sum_{j=1}^{n}\left[a_{j}(t, x, D z, z ; u)-a_{j}\left(t, x, D z_{0}, z_{0} ; u\right)\right]\left(D_{j} z-D_{j} z_{0}\right) d x \\
& +\int_{\Omega}\left[a_{0}(t, x, D z, z ; u, z)-a_{0}\left(t, x, D z_{0}, z_{0} ; u, z_{0}\right)\right]\left(z-z_{0}\right) d x \\
& =\int_{\Omega}\left[F_{2}(t, x ; u)-F_{2}^{\infty}\left(x, w_{0}\right)\right]\left(z-z_{0}\right) d x \\
& -\int_{\Omega} \sum_{j=1}^{n}\left[a_{j}\left(t, x, D z_{0}, z_{0} ; u\right)-a_{j}^{\infty}\left(x, D z_{0}, z_{0} ; w_{0}\right)\right]\left(D_{j} z-D_{j} z_{0}\right) d x \\
& -\int_{\Omega}\left[a_{0}\left(t, x, D z_{0}, z_{0} ; u, z_{0}\right)-a_{0}^{\infty}\left(t, x, D z_{0}, z_{0} ; w_{0}, z_{0}\right)\right]\left(z-z_{0}\right) d x \\
& \leq\left\|F_{2}(t, x ; u)-F_{2}^{\infty}\left(x, w_{0}\right)\right\|_{L^{q}(\Omega)}\left\|z(t)-z_{0}\right\|_{L^{p}(\Omega)} \\
& +\sum_{j=1}^{n}\left\|a_{j}\left(t, x, D z_{0}, z_{0} ; u\right)-a_{j}^{\infty}\left(x, D z_{0}, z_{0} ; w_{0}\right)\right\|_{L^{q}(\Omega)}\left\|D_{j} z(t)-D_{j} z_{0}\right\|_{L^{p}(\Omega)} \\
& +\left\|a_{0}\left(t, x, D z_{0}, z_{0} ; u, z_{0}\right)-a_{0}^{\infty}\left(x, D z_{0}, z_{0} ; w_{0}, z_{0}\right)\right\|_{L^{q}(\Omega)}\left\|z(t)-z_{0}\right\|_{L^{p}(\Omega)} .
\end{aligned}
$$

Since $p>1$ and $\|u\|_{L^{2}\left(Q_{t} \backslash Q_{t-a}\right)}^{\beta}$ is bounded for $t \in(0, \infty)$ by (3.9), thus (3.3)-(3.5), (3.18) imply (3.12).

Remark 3.3. Assume that the inequalities (3.3)-(3.5) hold such that for $j=1, \ldots, n$

$$
\begin{aligned}
\left|a_{j}(t, x, \xi ; u)-a_{j}^{\infty}(x, \xi ; u)\right| & \leq \text { const }\left[\left\|u(t)-w_{0}\right\|_{L^{p}\left(Q_{t} \backslash Q_{t-a}\right)}+\eta(t)\right]\left[1+|\xi|^{p-1}\right], \\
\left|a_{0}\left(t, x, \xi ; u, z_{0}\right)-a_{0}^{\infty}(x, \xi ; u, z)\right| & \leq \text { const }\left[\left\|u(t)-w_{0}\right\|_{L^{p}\left(Q_{t} \backslash Q_{t-a}\right)}+\eta(t)\right]\left[1+|\xi|^{p-1}\right], \\
\left|F_{2}(t, x ; u)-F_{2}^{\infty}\left(x ; w_{0}\right)\right| & \leq \text { const }\left[\left\|u(t)-w_{0}\right\|_{L^{p}\left(q_{t} \backslash Q_{t-a}\right)}+\eta(t)\right] .
\end{aligned}
$$

Then

$$
\left\|z(t)-z_{0}\right\|_{V_{2}}^{p-1} \leq \mathrm{const}\left[\mathrm{e}^{-c_{1} t}+\eta(t)\right], \quad t>0
$$


The above inequalities are satisfied e.g. if

$$
\begin{aligned}
a_{j}(t, x, \xi ; u) & =g_{j}(x, \xi)\left[1+\int_{t-a}^{t}|u(\tau, x)| d \tau+\eta(t)\right], \quad j=1, \ldots, n \\
a_{0}(t, x, \xi ; u, z) & =g_{0}(x, \xi)\left[1+\int_{t-a}^{t}|u(\tau, x)| d \tau+\eta(t)\right]
\end{aligned}
$$

where

$$
\begin{gathered}
\left|g_{j}(x, \xi)\right| \leq \operatorname{const}\left[|\xi|^{p-1}+\tilde{g}(x)\right], \quad \tilde{g} \in L^{q}(\Omega), \quad \eta \geq 0, \lim _{\infty} \eta=0, \\
\sum_{j=0}^{n}\left[g_{j}(x, \xi)-g_{j}\left(x, \xi^{\star}\right)\right]\left(\xi_{j}-\xi_{j}^{\star}\right) \geq c_{2}\left|\xi-\xi^{\star}\right|^{p}
\end{gathered}
$$

with some constant $c_{2}>0$.

\section{Acknowledgements}

This work was supported by the Hungarian National Foundation for Scientific Research under grant OTKA K 81403.

\section{References}

[1] R. A. Adams, Sobolev spaces, Academic Press, New York-San Francisco-London, 1975. MR0450957

[2] J. Berkovits, V. Mustonen, Topological degree for perturbations of linear maximal monotone mappings and applications to a class of parabolic problems, Rend. Mat. Appl. (7) 12(1992), 597-621. MR1205967

[3] Á. BesenYei, On nonlinear systems containing nonlocal terms, PhD thesis, Eötvös Loránd University, Budapest, 2008.

[4] S. CINCA, Diffusion und Transport in porösen Medien bei veränderlichen Porosität (in German), diploma thesis, Univ. Heidelberg, 2000.

[5] J. L. Lions, Quelques méthodes de résolution des problemes aux limites non linéaires (in French), Dunod; Gauthier-Villars, Paris, 1969. MR0259693

[6] J. D. Logan, M. R. Petersen, T. S. Shores, Numerical study of reaction-mineralogyporosity changes in porous media, Appl. Math. Comput. 127(2002), 149-164. MR1883122; url

[7] J. H. Merkin, D. J. Needham, B. D. Sleeman, A mathematical model for the spread of morphogens with density dependent chemosensitivity, Nonlinearity 18(2005), 2745-2773. MR2176957; url

[8] L. Simon, On some singular systems of parabolic functional equations, Math. Bohem. 135(2010), 123-132. MR2723079

[9] L. Simon, On singular systems of parabolic functional equations, Oper. Theory Adv. Appl. 216(2011), 317-330. MR2858877; url 
[10] L. Simon, Application of monotone type operators to parabolic and functional parabolic PDE's, in: Handbook of differential equations: evolutionary equations, Vol. 4, Elsevier, 2008, 267-321. MR2508168; url

[11] L. Simon, Semilinear hyperbolic functional equations, Banach Center Publ. 101(2014), 207-224. MR3288661; url

[12] L. Simon, On a system of nonlinear partial functional differential equations, Novi Sad J. Math. 45(2015), 253-265. MR3412060

[13] J. Wu, Theory and applications of partial functional differential equations, Applied Mathematical Sciences, Vol. 119, Springer-Verlag, 1996. MR1415838; url

[14] E. ZEIDLER, Nonlinear functional analysis and its applications II/A. Linear monotone operators, Springer-Verlag, New York, 1990. MR1033497; url

[15] E. ZEIDLER, Nonlinear functional analysis and its applications II/B. Linear monotone operators, Springer-Verlag, New York, 1990. MR1033498; url 